(C) 1984. The Genetical Society of Great Britain

\title{
MUTATIONAL LOAD AND THE ADVANTAGE OF SEX
}

\author{
D. P. E. DICKSON AND J. T. MANNING \\ Department of Science and Mathematics Studies, Faculty of Education and Extension \\ Studies, University of Liverpool, Liverpool L69 3BX
}

Received 13.ii.84

\section{SUMMARY}

\begin{abstract}
The mutational load for dominant deleterious mutations is $2 u$ in sexual populations (where $u$ is the mutation rate), but this reduces to between $2 u$ and $u$ when the selective disadvantage of the heterozygote is less than about 0.03 . In contrast, it is shown here that in the case of asexual population, the situation is different and the mutational load remains at $2 u$ even when the mutations are only very mildly deleterious in the heterozygote. As such mutations could be very common, sexually reproducing organisms may have a substantially smaller mutational load than asexual groups.
\end{abstract}

\section{INTRODUCTION}

A sexual population made up of equal numbers of males and females has a two-fold reproductive disadvantage compared to a population of parthenogenetic females (Maynard Smith, 1971; Manning, 1976a). Why, therefore, is asexual reproduction not more common? The answer could be that, while sex is indeed maladaptive, there is some obstacle to the origin of parthenogenetic females (see Williams, 1975 and Maynard Smith, 1978 for a discussion). An alternative explanation is that sexual progeny are in some way at least twice as fit as asexual offspring. This latter explanation, as well as being intrinsically more attractive, also explains the spread of sexual mutants within populations. The fitness advantage of sex, which must be at least two-fold, may have a number of components contributing to it.

Mutations, both advantageous and deleterious, could be an important factor in the widespread distribution of sex (Manning, 1976b). Sexual populations may accumulate favourable mutations more rapidly than asexual populations (Fisher, 1930; Maynard Smith, 1978; Manning 1983). In addition, and more importantly for short-term selective benefits, the load from harmful mutations may be lower in sexual populations than in asexual populations. This difference in mutational load could arise because of the ratchet mechanism in asexual populations (Muller, 1963; Felsenstein, 1974; Haigh, 1978; Manning, 1983), or because fitnesses are non-multiplicative (Kimura and Maruyama, 1966; Crow, 1970; Kondrashov, 1983), or for other reasons.

The purpose of this paper is to calculate the mutational load per gene for asexual populations and compare it with that for sexual populations. It is well known that the mutational load for dominant deleterious mutations in sexual populations is $2 u$, where $u$ is the mutation rate. However, when the selective disadvantage for the heterozygote becomes less than about 0.03 the mutational load decreases continuously from $2 u$ to $u$ (Kimura, 1961). It is shown here that in asexual populations with mutations which 
are only very mildly deleterious in the heterozygote (i.e., with a selective disadvantage of much less than 0.03 , but greater than the mutation rate, which might typically be $10^{-5}$ ), the mutational load remains at $2 u$, the same value as it has when the disadvantage is much greater.

\section{The Model}

Consider a diploid asexual population of $N$ individuals in which a normal gene $a$ mutates to a dominant allelomorph $A$ with a frequency $u$ per generation. The fitnesses of $a a, A a$ and $A A$ are 1, 1-hs and 1-s respectively, where $s$ is the selective disadvantage of the homozygote and $h s$ is the selective disadvantage of the heterozygote; $h$ is thus a measure of dominance. In the normal situation where $h s$, although possibly quite small, is greater than $u$, an equilibrium between mutation and selection will eventually be reached where the frequencies of individuals with $a a, A a$ and $A A$ are $1-x-y, x$ and $y$ respectively. (In the case where the selective disadvantage of the heterozygote $h s$ becomes less than the mutation rate $u$, the equilibrium situation is with no individuals with $a a$ and the following analysis is inappropriate.)

Once equilibrium has been established the numbers of individual eggs of each genotype in a generation are given by $N(1-x-y) ; N x ; N y$. After selection and mutation (ignoring the origin of $A A$ directly from $a a$ and back mutation from $A$ to $a$ as both being very unlikely) and restoring the total population to $N$ by multiplying by a scaling factor $\alpha$, the numbers of individuals of each genotype in the next generation are given by: $\alpha N(1-x-$ $y)(1-2 u) ; \alpha N[x(1-h s)(1-u)+(1-x-y) 2 u] ; \alpha N[y(1-s)+x(1-h s) u]$. At equilibrium, the numbers of individuals of each genotype in each generation must be the same and hence $\alpha=1 /(1-2 u)$. The mutational load $M$, is the fractional loss each generation, so that: $(1-M) \alpha=1$ and therefore $M=2 u$. Thus for the asexual case the mutational load is always $2 u$, unless $h s$ becomes less than $u$, when it decreases to $u$ as $h s$ decreases to zero. The same result can be obtained by extending the calculation of Crow (1970) to the present case of asexual diploids.

For sexual populations Haldane (1937) has shown the mutational load to be $2 u$ for dominant deleterious mutations. This result is valid if $h s$ lies between 1 and approximately 0.03 . However, when $h s$ becomes less than 0.03 Kimura (1961) has shown that the load is given by $u[1-\theta+\sqrt{\theta(2+\theta)}]$ where $\theta=s h^{2} /[2 u(1-2 h)]$. For example, if $u=10^{-5}, s=0.5$, and $h s=0.001$, this gives a mutational load of $1 \cdot 36 u$ for the sexual case compared with $2 u$ for the asexual case, a substantial difference.

The underlying reason for this difference lies in segregation, which occurs in the sexual population. When $A a$ individuals have a significant selective disadvantage the $A$ mutations are mainly eliminated from heterozygotes and this is true both for sexual and asexual populations. If the selective disadvantage is less, $A a$ individuals are more common and substantial numbers of homozygotes $(A A)$ can be produced. In asexual populations homozygotes can only be produced from heterozygotes by mutation, but in sexual populations $A A$ individuals can be produced both by mutation and by segregation which leads to an increase in the production of homozygotes as heterozygotes become more numerous. When this situation is reached many $A$ mutations are eliminated in pairs and this has the effect 
of reducing the mutational load. The value of 0.03 represents the critical value of the selective disadvantage of the heterozygote at which the behaviour in the sexual population changes. For a selective disadvantage above this the $\boldsymbol{A}$ mutations are mainly eliminated singly from heterozygotes, while for values below this the $\boldsymbol{A}$ mutations are often eliminated in pairs from the homozygotes. In asexual populations, in which there is no segregation, the situation is different and the elimination of the $A$ mutations mainly occurs singly in the heterozygotes, unless the selective disadvantage of the heterozygote is less than the mutation rate.

\section{Discussion}

The above model shows that the load from mutations which are only mildly deleterious in the heterozygote may be higher in asexual groups when compared to sexual populations. Unfortunately, we do not know how common such mutations are. Work with Drosophila (Mukai, 1964; Mukai, Chigusa, Mettler and Crow, 1972) has shown that mutations causing a small decrease in homozygous viability are much more common than lethal or semi-lethal mutations. In the heterozygote the selective disadvantage is small, i.e., often no larger than 3 per cent. However, most importantly for this model the average value of $h s$ may be considerably smaller than this because the procedure provides only a maximum estimate. That is, very many mutations with a disadvantage substantially less than 3 per cent could have been missed. For example, Crow and Kimura (1979) suggest the inclusion of these mutations may double the estimated mutation rate of the genome. If this is so segregation (i.e., sex), may lower the mutational load substantially.

\section{REFERENCES}

CROW. J. F. 1970. Genetic loads and the cost of natural selection. In Kojima, K. (ed.) Mathematical Topics in Population Genetics, Springer-Verlag, Berlin.

CROW, J. F. AND KIMURA, M. Efficiency of truncation selection. Proc. Nat. Acad. Sci. U.S.A., 76, 396-399.

FELSENSTEIN. J. 1974. The evolutionary advantage of recombination, Genetics, 78, 737-756. FISHER, R. A. 1930. The Genetical Theory of Natural Selection. Oxford University Press, Oxford. HAIGH. J. 1978. The accumulation of deleterious genes in a population-Muller's ratchet. Theor. Pop. Biol., 14, 251-267.

haldane, J. B. S. 1937. The effect of variation on fitness. Am. Nat. 71, 337-349.

KIMURA, M. 1961. Some calculations on the mutational load. Jap. J. Genetics, 36 Suppl., 179-189. KIMURA, M. AND MARUYAMA, T. 1966. The mutational load with epistatic gene interactions in fitness. Genetics, 54, 1337-1351.

KONDRASHOV, A. S. 1982. Selection against harmful mutations in large sexual and asexual populations. Genet. Res., 40, 325-332.

MANNING, J. T. 1976a. Gamete dimorphism and the cost of sexual reproduction: are they separate phenomena? J. Theor. Biol. 55, 393-395.

MANNING, J. T. 1976 b. Is sex maintained to facilitate or minimise mutational advance? Heredity, $36,351-357$.

MANNING, J. T. 1983. The consequences of mutation in multi-clonal asexual species. Heredity, 50, $15-19$.

MAYNARD SMITH, J. 1971. The origin and maintenance of sex. In Williams, G. C. (ed.) Group Selection, Aldine-Atherton, Chicago.

MAYNARD SMith, J. 1978. The Evolution of Sex. Cambridge University Press. 
MUKAI, T. 1964. The genetic structure of natural populations of Drosophila melanogaster: Spontaneous mutation rate of polygenes controlling viability. Genetics, 50, 1-19. MUKAI, T., CHIGUSA, S. J., METTLER, L. E. AND CROW, J. F. 1972. Mutation rate and dominance of genes affecting viability in Drosophila melanogaster. Genetics, 72, 335-355.

MULLER, H. J. 1964. The relation of recombination to mutational advance. Mut. Res., 1, 2-9. WILliamS, G. C. 1975. Sex and Evolution. Princeton University Press. 